\title{
Imaging of the Rotator Cuff and Biceps Tendon
}

\author{
Maj LJ King \\ MRCP,FRCR,RAMC*
}

\author{
JC Healy \\ MRCP,FRCR
}

\author{
Department of Diagnostic Radiology, Chelsea and Westminster Hospital,369 Fulham Road,London SW10 9NH \\ P Baird \\ FRCS
}

Department of Orthopaedics, Chelsea and Westminster Hospital,369 Fulham Road,London SW10 9NH

SUMMARY: Several different imaging techniques are available for evaluating the rotator cuff and biceps tendon. The common disorders of impingement, rotator cuff tears and biceps tendonitis are discussed along with the role which the various imaging modalities can play in establishing their diagnosis. Plain radiographs can be helpful particularly with a history of trauma but give limited information on the soft tissues. Ultrasound is a useful and inexpensive means of assessing the rotator cuff and biceps tendon but has a number of limitations and varying reports on its accuracy. Computed tomography (CT) is most helpful in the evaluation of shoulder trauma but gives limited information on the soft tissues. Magnetic resonance imaging (MRI) is an accurate imaging modality for evaluating the rotator cuff and biceps tendon, allowing visualisation of the soft tissues and the adjacent bony structures.

\section{Introduction}

Patients with rotator cuff pathology often present with classical signs and symptoms allowing an accurate clinical diagnosis to be made. Distinguishing clinically between rotator cuff pathology and other shoulder disorders such as biceps tendonitis, can be difficult however, and a variety of imaging techniques may be used in further evaluation of such cases. The imaging modalities available range from standard plain radiographs to state of the art MRI using intra-articular contrast and phased array surface coils. Each modality has a number of strengths and weaknesses in the evaluation of the rotator cuff and biceps tendon. The role of ultrasound and MRI is still evolving and their use in relation to shoulder arthroscopy is not universally agreed upon by surgeons and radiologists.

\section{Radiological Techniques \\ Plain Radiographs}

Conventional radiographs demonstrate the bony anatomy of the shoulder joint providing varying amounts of useful information in different clinical settings. They are a useful first line investigation in patients with rotator cuff symptoms and may demonstrate bony trauma, an anatomical variant or shoulder arthropathy. Plain radiographs give limited information regarding the soft tissue structures but in some cases may demonstrate soft tissue calcification or abnormal alignment of the shoulder joint implying a soft tissue disorder such as a cuff tear.

\section{Ultrasound}

Shoulder ultrasound is performed with a high frequency linear array transducer, and in the hands of an experienced operator is an accurate technique for detecting rotator cuff pathology. Ultrasound offers better spatial resolution than MRI and is a dynamic examination, which can be performed during shoulder movement. It benefits from being relatively inexpensive and not requiring the use of ionising radiation. Ultrasound can also be used to guide therapeutic injections around the shoulder tendons thus avoiding inadvertent intra-substance injection with the associated complication of tendon rupture. Drawbacks of ultrasound include the requirement for considerable operator expertise, blind areas and technical failure due to sub-optimal patients such as those with excess subcutaneous fat.

\section{Arthrography}

Arthrography is now used predominantly with either CT or MRI for visualising the glenoid labrum and joint capsule in cases of gleno-humeral instability. Prior to the advent of more sophisticated imaging techniques standard arthrography was used to identify full thickness rotator cuff tears by demonstrating the passage of contrast through the tendon defect into the sub acromial bursa. Iodinated contrast medium and air are injected into the shoulder joint for standard X-ray or CT arthrography, and saline or dilute intra-articular gadolinium are used for magnetic resonance arthrography.

Indirect magnetic resonance arthrography is a non-invasive alternative to the direct technique, which does not require an intra-articular injection. Following administration of intravenous paramagnetic contrast medium (gadolinium), the shoulder joint is exercised for a short period during which contrast diffuses into the joint cavity with resulting enhancement (1-3).

\section{Computed Tomography}

Helical CT provides an excellent depiction of bony anatomy in the axial plane which can then be manipulated to produce images in other planes, or to give surface shaded three dimensional reconstructions. Plain $\mathrm{CT}$ is better than MRI at delineating fractures (Fig 1) although MRI is sensitive to bone marrow oedema, which may occur following trauma. CT is also helpful in identifying loose bodies within the shoulder joint, but is of little use in the investigation of rotator cuff or biceps tendon pathology due to its relatively poor soft tissue contrast.

\section{Magnetic Resonance Imaging}

MRI has revolutionised shoulder joint imaging by providing multi-planar images with exceptional soft tissue contrast. This allows evaluation of rotator cuff degeneration, tears, structural abnormalities associated with impingement syndrome and disorders which may mimic pathology of the rotator cuff such as biceps tendonitis. MRI offers better contrast resolution than ultrasound, is a more reproducible technique and gives a global 


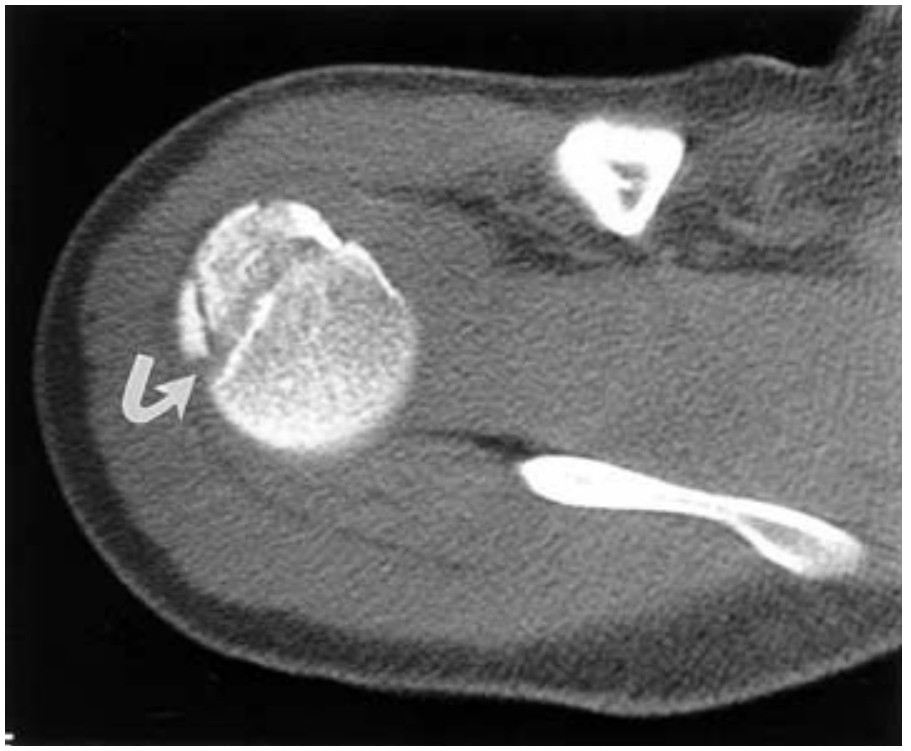

Fig 1. Comminuted fracture of the humeral neck. Axial CT image of the right shoulder demonstrates extension of the fracture into the greater tuberosity (curved arrow).

examination of the shoulder allowing evaluation of all the soft tissue and bony structures. Magnetic resonance arthrography can also be used to provide additional information and may clarify abnormalities seen on a standard MRI examination. A small number of patients will be unable to undergo MRI due to contraindications such as a cardiac pacemaker or because of claustrophobia which prevents completion of a satisfactory MRI scan in approximately 1 to $5 \%$ of patients (4-7).

\section{Anatomy of the Rotator Cuff and Biceps Tendon Rotator cuff}

The rotator cuff is formed by the tendons of 4 muscles: supraspinatus, infraspinatus, teres minor and subscapularis. The supraspinatus and infraspinatus tendons insert into the superior portion of the greater tuberosity, the teres minor tendon inserts into the posterior aspect of the greater tuberosity and the subscapularis tendon inserts medially into the lesser tuberosity. As well as contributing to shoulder movements the rotator cuff tendons centralise the humeral head within the glenoid and limit superior translation during abduction.

\section{Rotator interval}

The rotator interval is the space between the supraspinatus tendon and the superior border of the subscapularis tendon. It is formed from thin, elastic, membranous tissue reinforced by the coracohumeral ligament, superior glenohumeral ligament and the joint capsule.

\section{Coraco-acromial arch}

The coraco-acromial arch is comprised of the undersurface of the anterior third of the acromion, the coraco-acromial ligament and the acromio-clavicular joint. The coraco-acromial ligament is a triangular band, which originates from the lateral aspect of the coracoid and attaches to the acromion. The arch prevents superior ascent of the humeral head and is separated from the rotatorcuff by the subacromial/subdeltoid bursa, which serves as a gliding mechanism between these two structures.

\section{Long head of biceps}

The long head of biceps muscle flexes the arm and forearm, and assists in centralising and stabilising the shoulder joint. The tendon of the long head arises from the supraglenoid tubercle of the scapula and arcs over the humeral head before exiting the joint via an opening in the capsule above the bicipital groove in the hiatus between the subscapularis and supraspinatus tendons. Fibres of the biceps tendon contribute to the posterior and superior labrum forming the biceps-labral complex. The vertical extra-articular portion of long head of biceps lies in the bicipital groove between the lesser and greater tuberosities and is surrounded by a synovial sheath down to the level of the surgical neck of the humerus. This synovial sheath is continuous with the synovial space of the glenohumeral joint (8).

\section{Rotator Cuff and Biceps Tendon Pathology \\ Impingement Syndrome}

Impingement is due to painful entrapment of the supraspinatus tendon, subacromial-subdeltoid bursa and biceps tendon, between the humeral head and the coraco-acromial arch. The clinical manifestations of impingement syndrome are well documented often allowing precise diagnosis. The main role of imaging therefore is in defining the extent and cause of impingement (9) and differentiating from other shoulder disorders when the clinical features are unclear.

A variety of mechanisms have been proposed for impingement syndrome including hypovascularity of the supraspinatus tendon, mechanical wear, and acute trauma or repetitive microtrauma from overuse $(10,11)$. The distal supraspinatus tendon is vulnerable to injury as it runs in the confined space between the humerus and the coraco-acromial arch. Factors which may compromise the supraspinatous outlet resulting in impingement, include anterior acromial spurs and abnormal acromial or acromio-clavicular joint morphology. The varying shape of the acromion as seen on sagittal oblique MRI sections has been classified by Bigliani et al (12) as follows:

- type 1 (flat undersurface)

- type 2 (curved undersurface)

- type 3 (anterior hooking)

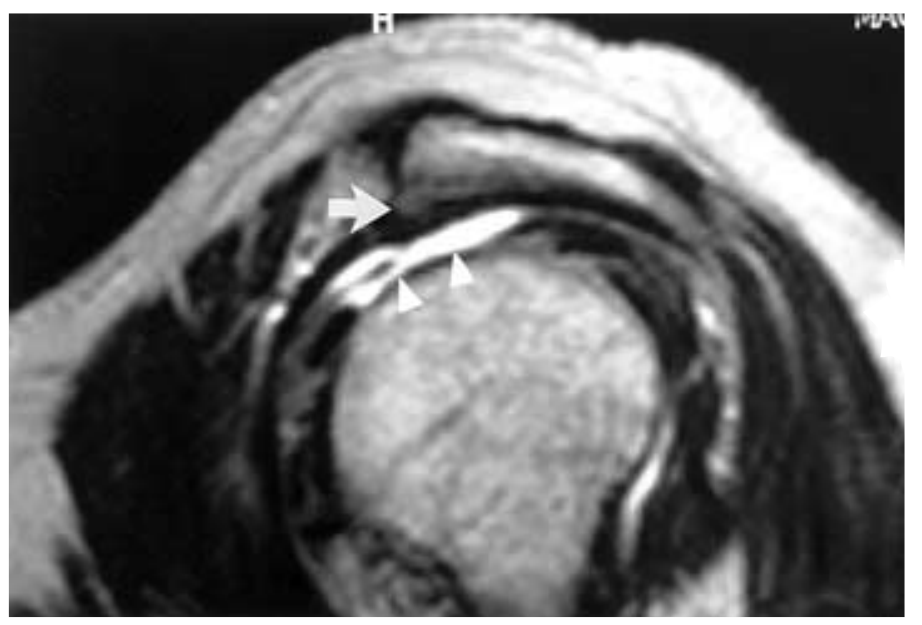

Fig 2. Sagittal T2 weighted magnetic resonance image of the right shoulder showing a Type 3 , anterior hooked acromion (arrow) in a patient with a full thickness supraspinatus tear (arrowheads).

Impingement is reported by some authors to be more common with a type 3 an anterior hooked acromion (13) (Fig 2). A lateral or anterior downward sloping of the acromion may also contribute to impingement by narrowing the supraspinatus outlet (14).

Other causes of impingement syndrome include secondary extrinsic impingement due to an unstable gleno humeral joint. Chronic shoulder instability is accompanied by weakening of the joint capsule and the gleno humeral ligaments, which are the 


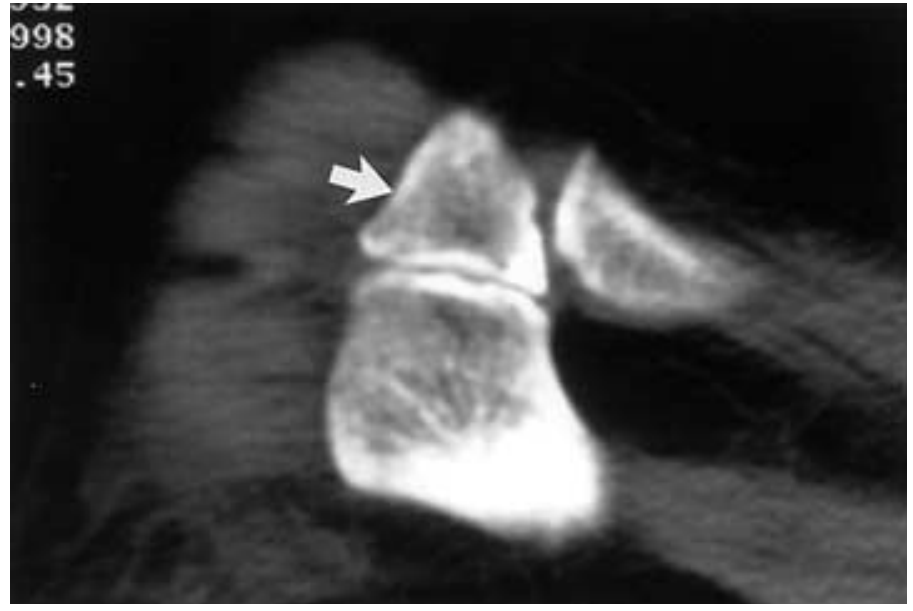

Fig 3. Sixty-five year old female with an incidental os acromiale demonstrated during evaluation of shoulder trauma. Axial CT image of the right shoulder demonstrates the unfused secondary ossification centre anteriorly (arrow).

static stabilisers of the gleno humeral joint. In this situation the dynamic stabilisers of the shoulder, namely the rotator cuff tendons, must play a greater role in preventing subluxation and dislocation. Excessive superior translation of the humeral head produces narrowing of the coraco-acromial outlet leading to undersurface impingement with eventual tendon degeneration and tear. The presence of an os acromiale, resulting from failure of fusion of a secondary ossification centre to the acromion (Fig 3 ), can also cause impingement. The deltoid muscle and coracoacromial ligament insert into the os acromiale, and traction by these structures may pull the unfused anterior fragment caudally resulting in dynamic impingement. In some cases, impingement is secondary to post-traumatic remodelling of the proximal humerus, for example, following a greater tuberosity fracture. An underlying mechanical cause is often not found however in patients with suspected impingement syndrome, and degenerative tendinopathy may be the primary pathology in these cases.

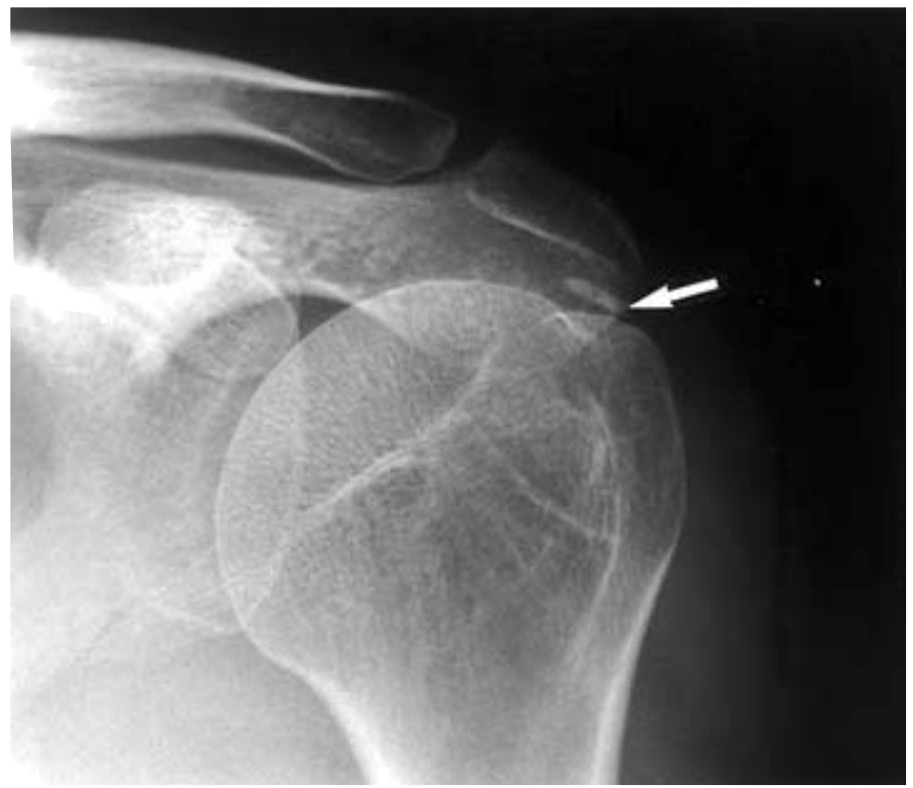

Fig 4. (a) Plain shoulder radiograph demonstrating a small area of calcification within the left supraspinatus tendon (arrow) in a patient with symptoms of rotator cuff impingement.

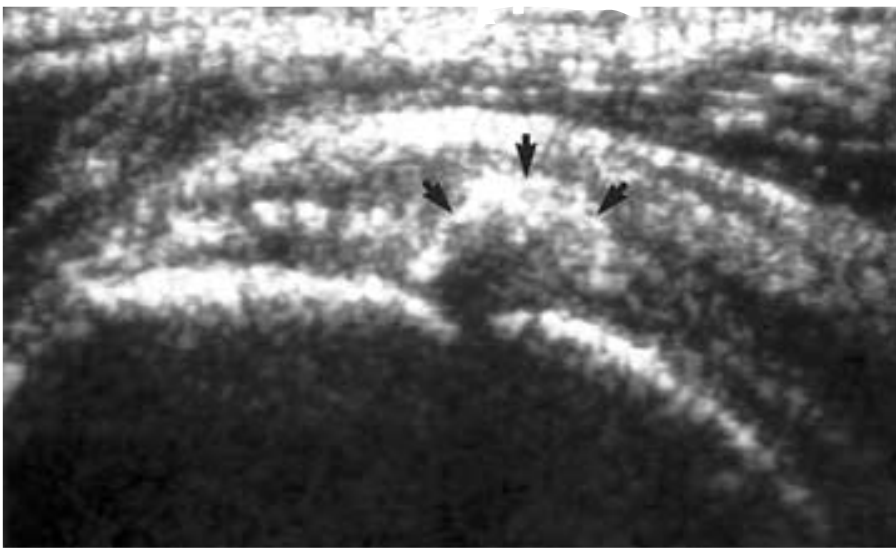

Fig 4. (b) Ultrasound of the right shoulder in a different patient with rotator cuff calcification demonstrates an echogenic region (arrows) with distal acoustic shadowing in the supraspinatus tendon.

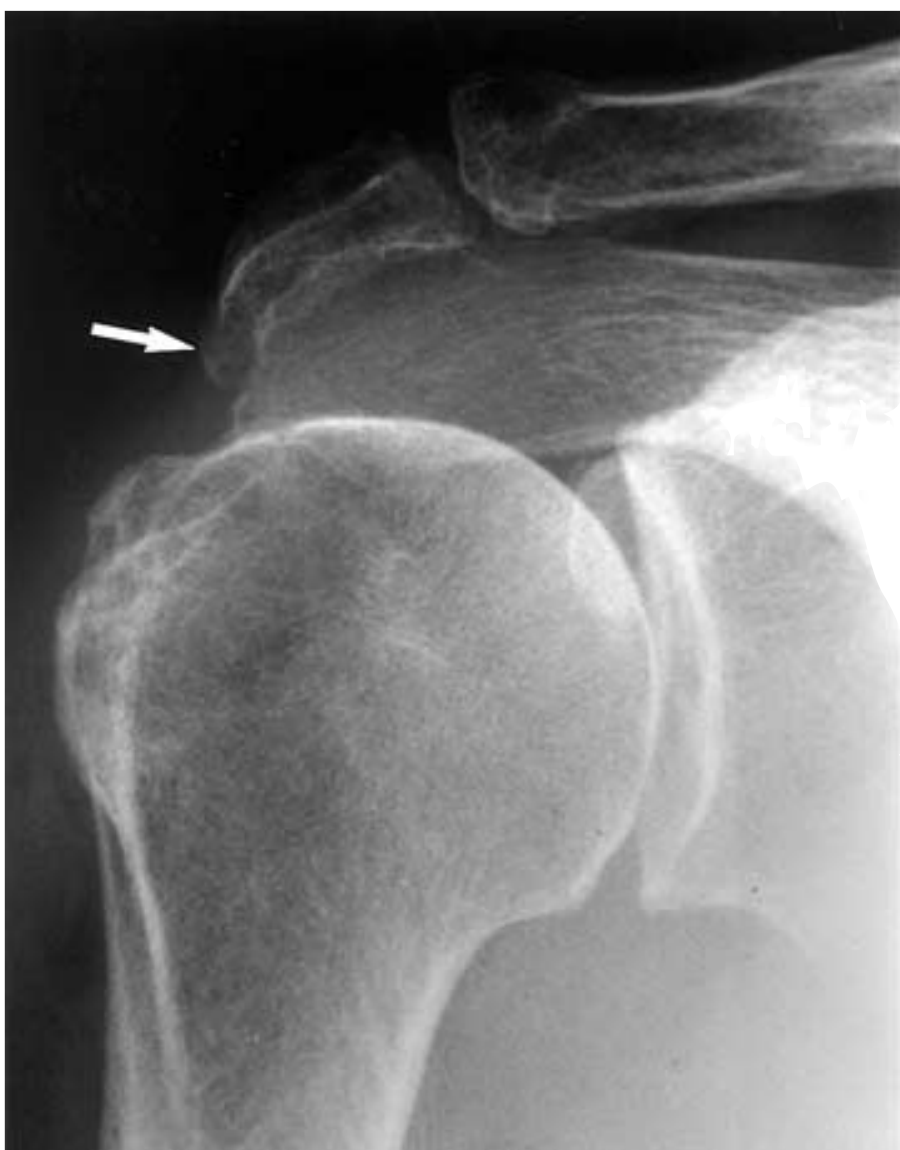

Fig 5. Plain radiograph of the right shoulder in an elderly female with symptoms of rotator cuff impingement. There are degenerative changes at the acromio-clavicular joint and a prominent subacromial osteophyte (arrow).

Plain radiographs of the shoulder are of limited value for evaluating the rotator cuff but may demonstrate calcification due to deposition of hydroxyapatite crystals secondary to a degenerative process. This most commonly occurs in the supraspinatus tendon, but can occur in any of the rotator cuff tendons and may be extremely painful requiring surgical debridement. Calcific deposits are best seen on plain films or ultrasound (Fig 4), as calcium has no signal on MRI and can be easily overlooked in the already low signal rotator cuff tendons. Plain radiographs may also demonstrate evidence of trauma, anterior subacromial osteophytes (Fig 5), degenerative change at 
the acromioclavicular joint and supraspinatus tendon insertion including sclerosis and cyst formation, or an anatomical variant such as an os acromiale. Superior subluxation of the humeral head with narrowing of the acromio-humeral distance may be apparent in some cases suggesting thinning or tearing of the supraspinatus tendon.

The MRI features of impingement are increased signal intensity in the rotator cuff tendons, subacromial bursitis and abnormal morphology of the coracoacromial arch including: the presence of subacrominal osteophytes, anterior hooking of the acromion, acromio-clavicular joint degeneration and a lateral or anterior downsloping acromion (12).

Ultrasound can be used to evaluate rotator cuff impingement by demonstrating fluid in the subacromial / subdeltoid bursa, identifying intra-substance tears or inflammatory change within the tendon, and showing tendon bunching or filling of the subdeltoid bursa during passive or active arm abduction.

Other less common forms of shoulder impingement are postero-superior glenoid impingement which is commonly seen in overhead throwing athletes (15) and coracohumeral impingement with encroachment upon the subscapularis tendon due to narrowing of the space between the coracoid process and the humeral head (Fig 6). The MRI appearances of coracohumeral impingement include thickening and signal inhomogeneity within the subscapularis tendon and fluid within the subcoracoid bursa (16).

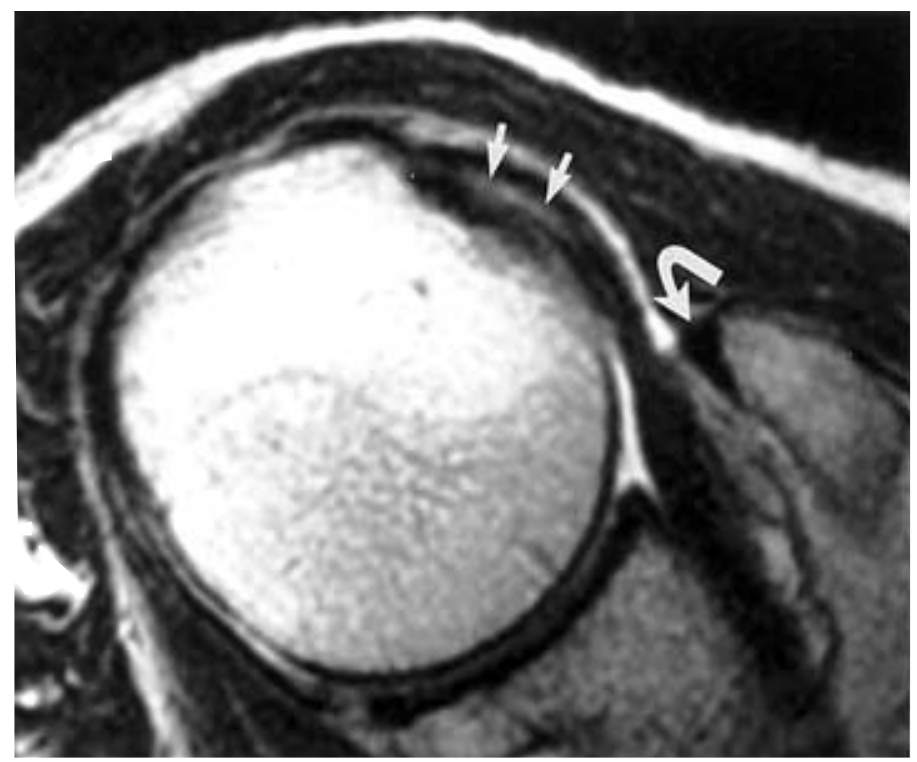

Fig 6. Male patient with subscapularis impingement. Axial T2 weighted magnetic resonance image of the right shoulder demonstrates a coracoid osteophyte (curved arrow) and high signal within the subscapularis tendon (arrows).

\section{Rotator Cuff Tears}

Tears of the rotator cuff can be classified as either partial or full thickness. Partial tears involve the articular or bursal surfaces with varying degrees of depth and extension into the tendon, or can be entirely intrasubstance in location not extending to the articular or bursal surfaces. Complete tears extend through the entire thickness of the rotator cuff and allow direct communication between the subacromial bursa and the glenohumeral joint. Tears most commonly involve the supraspinatus tendon, but extensive tears can involve at least two of the rotator cuff tendons.

Cuff tears have been attributed to a number of different aetiologies. Neer has postulated that $95 \%$ of rotator cuff tears result from chronic impingement (17). This theory is supported by Morrison and Bigliani who reported type 3 acromions in $80 \%$ of patients with rotator cuff tears (13). A similarly study by Epstein et al showed the presence of a hooked acromion in $62 \%$ of patients with full thickness tears compared to only $13 \%$ of normal controls (18). Other studies have found MR assessment of the acromial shape to be disappointing and have shown that MR readers vary in their ability to classify acromial morphology $(19,20)$. In contrast to Neer's hypothesis, some authors believe that ageing is the most important cause of rotator cuff tears followed by impingement, acute trauma, overuse and chronic inflammatory disease $(21,22)$.

It is currently thought that most partial rotator cuff tears involve the inferior articular surface of the tendon adjacent to its insertion $(23,25)$. These articular surface lesions may be due to tensile strength failure resulting from overuse. The less common bursal surface partial tears are more closely associated with impingement $(24,26)$. Cuff tears are common following greater tuberosity fractures and in a recent study 15/20 (75\%) patients with greater tuberosity fractures had abnormal rotator cuffs on MRI, including 9 patients $(45 \%)$ with full thickness supraspinatus tears (27).

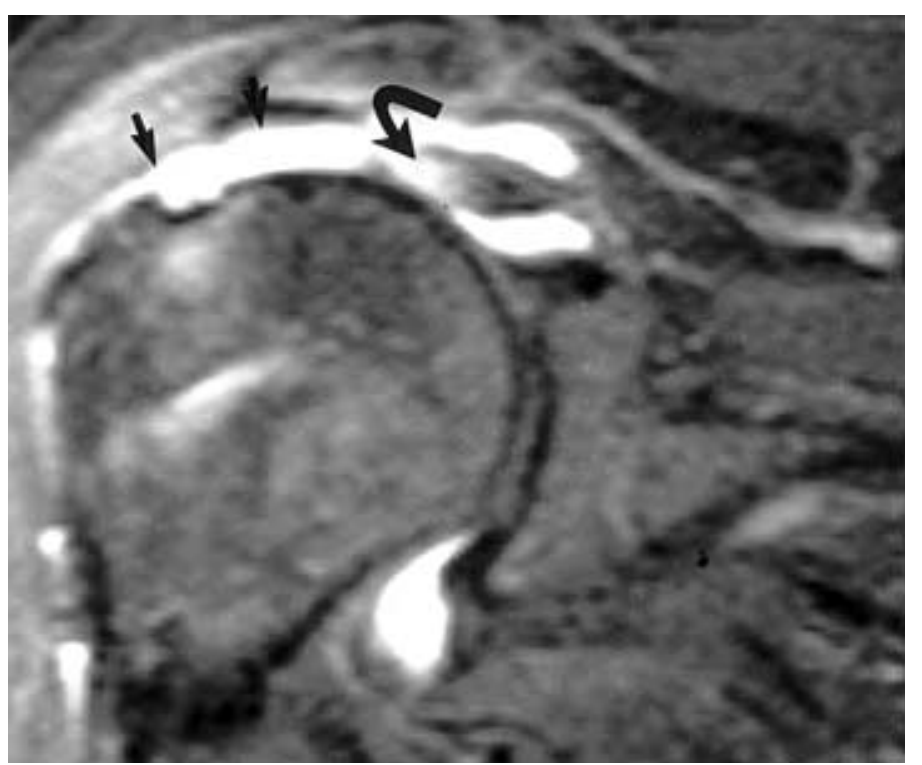

Fig 7. Coronal oblique STIR magnetic resonance image of the right shoulder in a 62 year old male showing disruption of the supraspinatus tendon with retraction of the proximal tendon (curved arrow) and high signal fluid in the subacromial/subdeltoid bursa (arrows).

MRI findings in full thickness rotator cuff tears include disruption of the normal, low signal intensity tendon by an area of high signal. Retraction of the torn tendon end may be seen with full thickness tears (Fig 7), which, if long standing, may be associated with diffuse muscle atrophy and fatty replacement. Fluid may also be seen in the subacromial / subdeltoid bursa especially in bursal surface partial thickness tears, or with full thickness tears when fluid can track through the defect from the gleno humeral joint. The presence of bursal fluid is not a definitive sign of a tear however as fluid can be present following trauma, in association with impingement or in inflammatory disorders such as rheumatoid arthritis. A small amount of fluid may also be present in asymptomatic patients (28). The sensitivity and specificity of MRI for identifying rotator cuff tears is high with reported accuracy rates of over $90 \%$ (29-33).

MR arthrography is reported to have increased sensitivity for the detection of rotator cuff tears compared with conventional 
MRI (34,35). Flannigan et al identified 11/14 (79\%) proven rotator cuff tears on MR arthrography compared to only $9 / 14$ (64\%) on conventional MRI (34). Hodler et al also compared MR arthrography with conventional MRI in 36 patients and found improved sensitivity and specificity values of $71 \%$ and $84 \%$ following intra-articular gadolinium compared to $41 \%$ and $79 \%$ on the conventional MRI (35). Interestingly however, the detection rate for rotator cuff tears utilising MR arthrography in these two studies is lower than that published in some studies with conventional MRI. This may be due to differences in equipment, sequences, patient selection and reader experience plus the utilisation of fat suppression sequences in more recent studies (33). A further increase in the conspicuity of cuff tears has been demonstrated by utilising fat suppressed imaging in conjunction with MR arthrography $(36,37)$ with sensitivity and specificity values of $100 \%$ reported in one series of 37 patients compared to $90 \%$ and $75 \%$ with non fat suppressed MR arthrography (36).

Superior delineation of rotator cuff tears has also been reported in a small group of patients using indirect MR arthrography $(2,3)$, with sensitivity and specificity values of $100 \%$ and $86 \%$ respectively. This technique may be useful in the further evaluation of patients with equivocal MRI examinations, without resorting to direct MR arthrography.

Tears can occur in other rotator cuff tendons, especially the subscapularis tendon, most commonly in association with a supraspinatus tear. Subscapularis tears can also accompany recurrent dislocation, particularly in the elderly, and occasionally occur in isolation. The features of subscapularis tears are similar to those described for the supraspinatus tendon and are often accompanied by medial subluxation of the biceps tendons. Tears also occur in the rotator interval between the supraspinatus and supscapularis tendons. These tears may extend into the subscapularis tendon and are often seen in association with acute dislocations of the gleno humeral joint.

Ultrasound can be used to diagnose rotator cuff tears by identifying similar features to those seen on MRI. Partial thickness tears show a mixed hyper and hypoechoic region or a hypoechoic discontinuity in the rotator cuff tendons. With a complete rupture (Fig 8), ultrasound may show disruption or absence of the tendon due to medial retraction under the acromion (38). Fluid may be seen filling the tendon defect or the gap may be filled by the deep surface of the deltoid muscle,

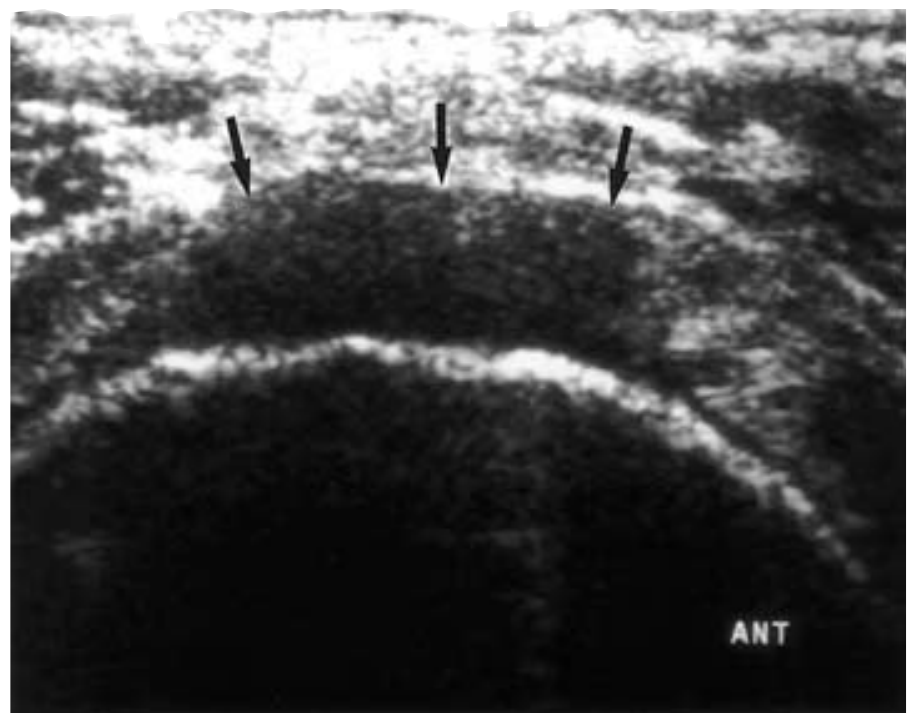

Fig 8. Coronal ultrasound image demonstrating a large hypeochoic region within the supraspinatus tendon consistent with a full thickness tear (arrows). which comes into close contact with the greater tuberosity (39). Abnormal morphology of the superior surface of the supraspinatus tendon can be demonstrated which appears concave rather than convex. The "cartilage interface sign" may also be identified, representing an echogenic reflection from the cartilage overlying the humeral head. There are blind areas which cannot be seen with ultrasound such as underneath the acromion, and this may be a problem if the patient is unable to sufficiently internally rotate the arm in order to expose the superior rotator cuff. It can also be difficult to get good views in patients with excessive overlying fat. Some authors have reported sensitivities and specificities above $90 \%$ for ultrasound in diagnosing full thickness rotator cuff tears (39-43). High sensitivity and specificity values of $93 \%$ and $94 \%$ have also been reported for identification of partial thickness tears with ultrasound (44). Other studies have concluded that there is a low level of reliability for ultrasound in detecting rotator cuff disease $(45,46)$. This variability has been attributed to a long learning curve in mastering the technique of shoulder ultrasound (43).

\section{Biceps Tenosynovitis}

Inflammation of the biceps tendon is frequently a degenerative process, occurring within the bicipital groove. It can occasionally result from trauma or may be associated with chronic inflammatory disorders such as rheumatoid arthritis.

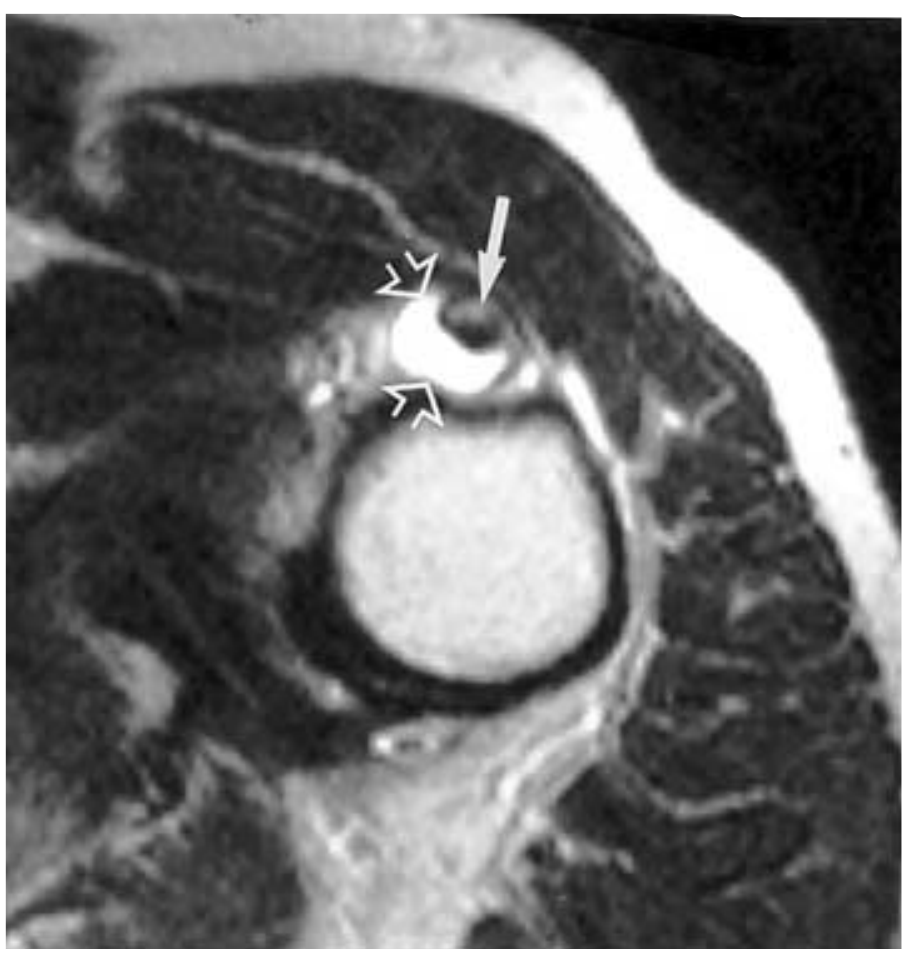

Fig 9. Axial T2 weighted MR image showing high signal in the biceps tendon (arrow) and high signal fluid within the tendon sheath (open arrows) due to biceps tendonitis.

MRI appearances commonly seen in the early phase of tendinosis include tendon thickening, diffuse intermediate signal within the tendon substance, synovial thickening or adhesions and fluid within the biceps tendon sheath as it lies in the bicipital groove (Fig 9). The biceps tendon sheath however is in direct continuity with the glenohumeral joint. Presence of fluid within the sheath is thus a non-specific finding and may be secondary to glenohumeral pathology. A small amount of fluid may also be present in normal individuals $(47,48)$.

The MRI findings in late tendinosis are a thin and diffusely attenutated tendon with diffuse intermediate signal and irregularity or fraying. If there is marked inflammation within the 


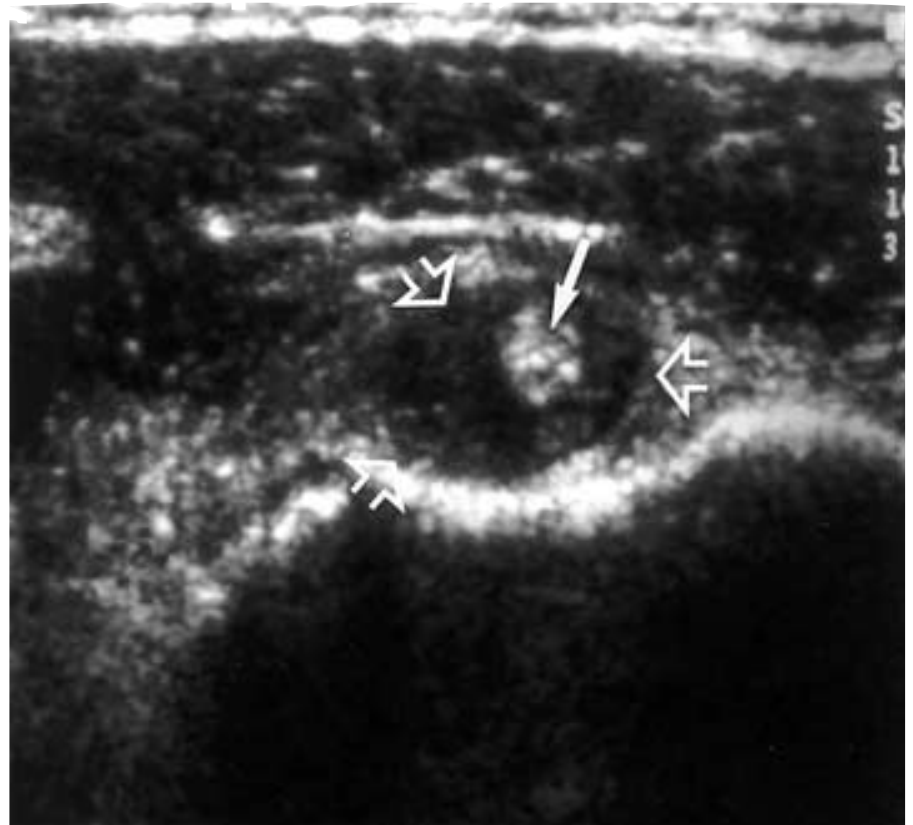

Fig 10. Transverse ultrasound image of the biceps tendon from a patient with chronic tendonitis. There is increased echogenicity within the tendon (arrow) which is surrounded by hypoechoic fluid and echogenic pannus (open arrows).

tendon sheath such as in rheumatoid arthritis, intermediate signal pannus may also be seen around the tendon.

Ultrasound is a useful technique for evaluating biceps tenosynovitis (Fig 10). Anechoic fluid can be demonstrated around the swollen tendon, which may itself be of low echogenicity. Echogenic, hypervascular pannus may also be seen using colour doppler ultrasound.

\section{Conclusion}

Imaging of the shoulder can be a helpful adjunct to clinical assessment, in patients with suspected rotator cuff or biceps tendon disorders. Plain radiographs are a useful first line investigation particularly if there is a history of trauma and may give information, which can assist in the interpretation of more modern imaging techniques such as ultrasound and MRI. Ultrasound is an inexpensive technique, which allows dynamic evaluation of the shoulder and in experienced hands is sufficiently accurate for it to be used as a screening tool for suspected rotator cuff or biceps tendon pathology. MRI gives the best global view of the shoulder and because of its superior soft tissue contrast elegantly demonstrates rotator cuff and biceps tendon pathology, although the choice of imaging modality in individual cases will also depend on the local availability and expertise.

\section{REFERENCES}

1. Winalski CS, Weissman BN, Aliabadi P, Wright RJ, ShortKrofF S, Sledge CB. Intravenous Gd-DTPA enhancement of joint fluid: a less invasive alternative for MR arthrography. Radiology 1991; 181: 304.

2. VAhlensieck M, Peterfy CG, Wischer T, et al., Indirect MR Arthrography: Optimization and Clinical Applications. Radiology 1996; 200:249-254.

3. VAHLENSIECK M, SOMMER T. Indirect MR arthrography of the shoulder. An alternative to direct MR arthrography? Radiologie 1996; 36(12):960-965.

4. FlaherTy JA, Hoskinson K. Emotional Distress during Magnetic Resonance Imaging. NEJM 1989; 320(7):467-468.

5. AVRAHAMI E. Panic Attacks During MR Imaging: Treatment with IV Diazepam. AJNR 1990; 11:833-835.

6. DANTEndorfer K, Wimberger D, Katschnig H, IMHOFF H. Claustrophobia in MRI scanners. Lancet 1991; 338:761-762.

7. Mackenzie R, Sims C, Owens RG, Dixon AK. Patient's Perceptions of Magnetic Resonance Imaging. Clin Radiol 1995; 50:137-143.

8. Klug JD, Moore SL. MR Imaging of the biceps muscletendon complex. Magn Reson Imaging Clin North Am 1997; 5:755.

9. RESNICK D. Shoulder Imaging. Magn Reson Imaging Clin North Am 1997; 5(4):663-664.

10. RATHBUn JB, MACNAB I. The microvascular pattern of the rotator cuff. J Bone Joint Surg 1970; 52:540.

11. BigLiAni LU, Morrison DS. Subacromial impingement syndrome. In: Dee R, ed. Principles of Orthopaedic Practice. New York: McGraw-Hill 1989:627.

12. Bigliani LU, MORRISON DS, APRIL EW. The morphology of the acromion and its relationship to rotator cuff tears. Orthop Trans 1986; 10: 228.

13. MoRrison DS, Bigliani LU. The clinical significances of variations in acromial morphology. Orthop Trans 1987; 11:234.

14. Banas MP, Miller RJ, TotTerman S. Relationship between the lateral acromial angle and rotator cuff disease. J Shoulder Elbow Surg 1995; 4:454-461.

15. Jobe CM. Posterior superior glenoid impingement: expanded spectrum. Arthroscopy 1995; 11:530.

16. BonutTi PM, Norfray JF, Friedman RJ, Genez BM. Kinematic MRI of the shoulder. J Comput Assist Tomogr 1993; 17:666.

17. NEER CS. Anterior acromioplasty for the chronic impingement syndrome a preliminary report. $J$ Bone Joint Surg (Am) 1972; 54:41.

18. Ebstein RE, Schweitzer ME, Frieman BG, Fenlin JM, Mitchell DG. Hooked Acromion: Prevalence on MR Images of Painful Shoulders. Radiology 1993; 1187:479-481.

19. HaYgood TM, LANGlotz CP, KNEEland BJ, et al. Categorization of acromial shape: Interobserver variability with MR imaging and conventional radiography. AJR 1994; 162:1377-1382.

20. PeH WCG, FARMER THR, TOTTY WG. Acromial arch shape: Assessment with MR imaging. Radiology 1995; 195:501505.

21. Ozaki J, Fukimoto S, Nakagawa Y, Masuhara K, Tamai S. Tears of the Rotator cuff. J Bone Joint Surg (Am) 1988; 70:1224-1230.

22. Ogata S, Uhthoff HK. Acromial enthesopathy and rotator cuff tear. A Radiologic and histologic postmortem investigation of the coarcoacromial arch. Clin Orthop 1990; 254:39.

23. FuKUda H, MiKasa M, Ogawa K, et al. The partial thickness tear of the rotator cuff. Orthop Trans 1983; 7:137.

24. TYSON LL, CRUES J. Pathogenesis of rotator cuff disorders. In: Rafii M ed. Magentic resonance imaging. Magn Reson Imaging Clin North Am 1993; 1:37.

25. UhthofF AK, SARKAR K. Classification and definition of tendinopathies. Clin Sports Med 1991; 10:707.

26. Ogata S, Uhthoff HK. Acromial enthesopathy and rotator cuff tear: a radiologic an histologic postmortem investigation of the coracoacromial arch. Clin Orthop 1990; 254:39.

27. MCCARThy DM, FEldman F, Staron RB. Rotator Cuff Injury Associated with Greater Tuberosity Fractures. Presented at RSNA 1997.

28. RESNIK D, ed. Internal derangements of joints. In: Diagnosis of bone and joint disorders, 3rd ed. Philadelphia: WB Saunders, 1995. 
29. Zlatkin MB, IANNOTTI JP, RoBERTS MC, et al. Rotator cuff tears: diagnostic performance of MR imaging. Radiology 1989; 172:223.

30. IANNOtTI JP, Zlatkin MB, Esterhai JL, Kressel HY, DALINKA MK, SPINDLER KP. Magnetic resonance imaging of the shoulder: sensitivity, specificity and predictive value. $J$ Bone Joint Surg (Am) 1991; 73:17.

31. BuRK DL JR, KARASICK D, KURTZ AB, et al. Rotator cuff tears; prospective comparison of the MR imaging with arthroscopy, sonography and surgery. AJR 1989; 153:81.

32. RAFII M, FiroOzNIA H, SHERMAN O, et al. Rotator cuff lesions: signal patterns at MR imaging. Radiology 1990; 177:817.

33. QuinN SF, Sheley RC, Demlow TH, SzUMowsKi J. Rotator cuff tendon tears: evaluation with fat suppressed MR imaging with arthroscopic correlation in 100 patients. Radiology 1995; 195:497.

34. Flannigan B, Kursunoglu-Brahme S, Karzel R, Del PIZZO W, RESNICK D. MR arthrography of the shoulder: comparison with conventional MR imaging. AJR 1990; 155(4):829-832.

35. Hodler J, Kursunoglu-Brahme S, Snydre SJ, et al., Rotator Cuff Disease: Assessment with MR Arthrography versus Standard MR Imaging in 36 Patients with Arthroscopic Confirmation. Radiology 1992; 182:431-436.

36. Palmer WE, Brown JH, Rosenthal DI. Rotator Cuff: Evaluation with Fat-suppressed MR Arthrography. Radiology 1993; 188:683-687.

37. Funke M, Kapka L, Vosshenrich R, OEstmann JW, GRABBE E. MR arthrography in the diagnosis of rotator cuff tears. Standard spin-echo alone or with fat suppression? Acta Radiol 1996; 37(5):627-632.
38. ChHEM RK, KaPlan PA, Dussault RG. Ultrasonography of the musculoskeletal system. Radiol Clin North Am 1994; 32(2):275-289.

39. Middleton WD, Reinus WR, Totty WG, Melson CL, MURPHY WA. Ultrasonographic evaluation of the rotator cuff and biceps tendon. J Bone Joint Surg (Am) 1986; 68:440-450.

40. Crass JR, Craig EV, FeInBERG SB. Ultrasonography of rotator cuff tears: A review of 500 diagnostic studies. J Clin Ultrasound 1988; 16:13-327.

41. Mack LA, Gannon MK, Kilcoyne RF. Sonographic evaluation of the rotator cuff: accuracy in patients without prior surgery. Clin Orthop 1988; 234:21-27.

42. FuRTSCHEGGER A, RESH H. Value of ultrasonography in preoperative diagnosis of rotator cuff tears and postoperative follow up. Eur J Radiol 1988; 8:69-75.

43. Mack LA, Nyberg DA, Matsen FA III. Sonographic evaluation of the rotator cuff. Radio Clin North Am 1988: 26:161-177.

44. Van Holsbeeck MT, Kolowich PA, Eyler WR, et al., US Depiction of Partial-Thickness Tear of the Rotator Cuff. Radiology 1995; 197:443-446.

45. Brandt TD, CARdone BW, GRANT TH, et al: Rotator cuff sonography: A reassessment. Radiology 1989; 173:323-327.

46. Miller CL, Karasick D, Murtz AB, et al: Limited sensitivity of ultrasound for the detection of rotator cuff tears. Skeletal Radiol 1989; 18:179-183.

47. Rafi M. Shoulder. In: Firooznia H, Golimbu C, Rafi M, eds: MRI and CT of the Musculoskeletal System. St. Louis, CV Mosby, 1990, pp468, 526-527.

48. VAN LEERSUM M, SCHWEITZER ME. Magnetic resonance imaging of the biceps complex. Magn Reson Imaging Clin North Am 1993; 1:77-86. 